\title{
Manejo quirúrgico de la luxación acromioclavicular grado III con sistema de anclaje doble botón
}

\author{
Acromioclavicular dislocation management with use of Tight \\ Rope luxation acromioclavicular con Tight Rope
}

\author{
Gaytán-Fernández S,* Blanco-Ochoa LC,* Barragán-Hervella RG,* \\ Montiel-Jarquín AJ, ${ }^{\ddagger}$ Sánchez-Durán MA,* García-Galicia A*
}

Unidad Médica de Alta Especialidad, Hospital de Traumatología y Ortopedia de Puebla del Instituto Mexicano del Seguro Social.

RESUMEN. Introducción: La articulación acromioclavicular es parte importante del complejo articular del hombro, formada por el extremo lateral de la clavícula y el borde medial del acromion. Tiene un alto índice de lesión en grados asociados a actividad física y accidentes laborales. Predomina en jóvenes laboralmente activos. Objetivo: Analizar la funcionalidad a un año en pacientes con luxación acromioclavicular grado III, operados con sistema anclaje doble botón. Material y métodos: Estudio descriptivo, prospectivo, longitudinal, unicéntrico, durante Marzo de 2015 a Julio de 2016, en pacientes con luxación acromioclavicular grado III en el Hospital de Traumatología y Ortopedia de Puebla. Se incluyeron pacientes con edad de 18 a 45 años, trabajadores, con evolución mayor de siete días. La estadística fue descriptiva. Resultados: Fueron 17 pacientes; 14 (82.4\%) hombres y tres (17.6\%) mujeres. Edad promedio: 29.5 (19 a 44 años) \pm 7.475 años; nueve (52.9\%) pacientes en el hombro derecho y ocho (47.1\%) en el izquierdo. Promedio de evolución: 2.88 (1 a 7) \pm 2.147 días. Incapacidad promedio: 42.06 días. Conclusiones: La reducción abierta y estabilización dinámica mediante el sistema anclaje doble botón (Tight Rope) ofrece estancia intrahospitalaria corta, poco tiempo de incapacidad, reintegración rápida a la actividad laboral y resultados funcionales adecuados a un año de evolución.

Palabras clave: Tratamiento, luxación acromioclavicular, anclaje doble botón.
ABSTRACT. Introduction: The acromioclavicular joint, important part of the shoulder complex is formed by clavicular lateral end and acromial medial border, with high index of injury in different grades, associated to physical activity and work accidents, most commonly in active young adults. Objective: To analyze functional outcomes in patients with acromioclavicular dislocation with use of tight rope in one year. Material and methods: Descriptive, prospective, longitudinal, unicentric study, from March 2015 and July 2016 in post-operated patients with diagnosis of acromioclavicular dislocation grade III in Hospital de Traumatología y Ortopedia de Puebla. Patients aged 18 to 45 years were included, active workers, with evolution time $<7$ days. Results: 17 patients with diagnosis of acromioclavicular dislocation, fourteen (82.4\%) male and 3 (17.6\%) female. Average age: 29.5 (19 to 44 years) \pm 7.475 years; lesion occurred 9 (52.9\%) patients in right shoulder and $8(47.1 \%)$ in left side. Average time of the injury: 2.88 $(1-7) \pm 2,147$ days. Average inability granted: 42.06 days. Conclusions: The open reduction technique and dynamic stabilization of the joint with Tight Rope offers short hospital stay, short time of inability and rapid reintegration to work activity, at 1 year of evolution.

Keywords: Management, acromioclavicular dislocation, tight rope.

\section{Nivel de evidencia: IV}

* Unidad Médica de Alta Especialidad, Hospital de Traumatología y Ortopedia de Puebla.

₹ Unidad Médica de Alta Especialidad, Hospital de Especialidades de Puebla.

Instituto Mexicano del Seguro Social.

Dirección para correspondencia:

Dr. Arturo García-Galicia

Jefatura de División de Investigación, Unidad Médica de Alta Especialidad, Hospital de Traumatología

y Ortopedia de Puebla del Instituto Mexicano del Seguro Social.

Diagonal Defensores de la República esquina 6 poniente S/N, Col. Amor, CP. 72140, Puebla, Puebla.

Tel: +52 222 2493099, ext. 208.

E-mail: arturo.garciaga@imss.gob.mx; neurogarciagalicia@yahoo.com.mx

Este artículo puede ser consultado en versión completa en: www.medigraphic.com/actaortopedica 


\section{Introducción}

La articulación acromioclavicular forma una parte importante del complejo articular del hombro, su movilidad es limitada. Sus lesiones son frecuentes, constituyen de 45 a $60 \%$ de todas las luxaciones. ${ }^{1}$

Existen varias clasificaciones para esta patología. La principal fue creada por Rockwood y colaboradores en 1984, y divide las lesiones en VI grados. ${ }^{1}$

El tratamiento ha sido discutido desde los tiempos de Hipócrates (460-377 a.C.). ${ }^{1}$

La mayoría de los autores coinciden en que el manejo de las lesiones grado I y II es conservador, y quirúrgico para los grados IV, V y VI. El manejo de las lesiones grado III ha sido controversial, ${ }^{1,2,3,4,5}$ se han implementado múltiples técnicas tanto conservadoras como quirúrgicas. ${ }^{6,7}$

Las técnicas quirúrgicas para el manejo de las lesiones grado III se dividen en articulares, que consisten en colocar un implante injerto, sutura o realizar alguna técnica relacionada con la articulación acromioclavicular; y extraarticulares, que utilizan métodos distintos con fijación a la apófisis coracoides. Anteriormente, se empleaba la reducción y estabilización estática de la articulación; sin embargo, demostró una tasa de éxito baja, debido a que los pacientes presentaban rigidez y fallas en los implantes. Debido a esto, se empezaron a emplear técnicas con estabilización dinámica que usan injertos, suturas y anclas mediante cirugía artroscópica y abierta, con resultados mejores que los de la estabilización estática. $8,9,10,11,12,13,14$

En nuestro hospital, según cifras del Sistema de Información Médico Operativo (SIMO) del Instituto Mexicano del Seguro Social, año 2016, la luxación de hombro tuvo una tasa de incidencia de 57.42 casos/10,000 egresos hospitalarios, así la luxación acromioclavicular grado III, IV, V y VI fue de 11.15/1,000 pacientes hospitalizados; se desconocen los tiempos de incapacidad laboral y de incorporación de los trabajadores a sus actividades. También se utilizan otras técnicas quirúrgicas para su manejo, como la técnica de Mumford, en la que se hace una resección distal de la clavícula con resultados no satisfactorios, por lo que se decidió realizar este es-



Figura 1: Sistema de anclaje de doble botón.

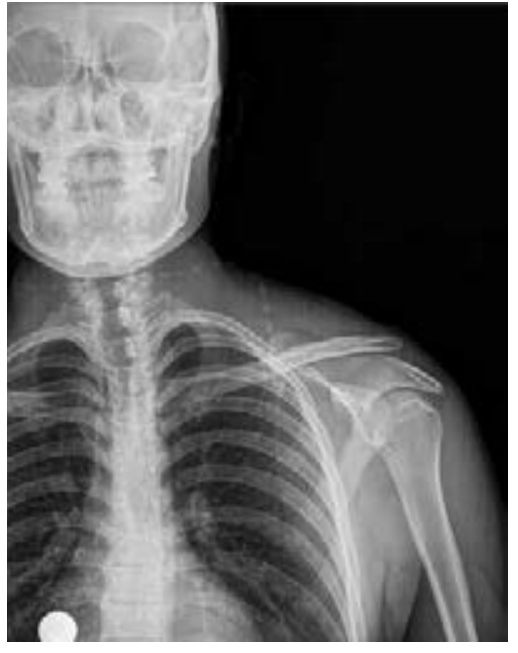

Figura 2:

Paciente con subluxación acromioclavicular grado III.

tudio, cuyo objetivo es analizar los resultados funcionales de la colocación del sistema de anclaje doble botón TighRope (Arthrex Inc., Naples Florida USA) como implante para el tratamiento de la luxación acromioclavicular grado III, en población joven laboralmente activa (Figura 1).

\section{Material y métodos}

Este proyecto fue autorizado por el Comité Local de Investigación y Ética en Investigación en Salud 2015 del Instituto Mexicano del Seguro Social, con número de registro nacional R-2017-2015-3. Todos los pacientes firmaron carta de consentimiento informado por escrito, y en todo momento se conservó el anonimato de los participantes y el manejo confidencial de su información.

Estudio descriptivo, prospectivo, longitudinal y unicéntrico realizado en pacientes con luxación acromioclavicular grado III, manejados quirúrgicamente con sistema de anclaje doble botón, en la Unidad Médica de Alta Especialidad, Hospital de Traumatología y Ortopedia de Puebla, durante Marzo de 2015 a Julio de 2016.

Se incluyeron pacientes de 18 a 45 años, de ambos géneros, laboralmente activos, con lesión unilateral, incidentes, de siete o menos días de evolución, con luxación acromioclavicular grado III. Se excluyeron pacientes que presentaron lesiones traumáticas como fracturas en otros sitios distintos y aquéllos con lesión de la cintura escapular asociada. Los pacientes eliminados fueron quienes no acudieron a seguimiento, que perdieron su afiliación institucional y los que fallecieron durante su seguimiento. El diagnóstico fue realizado con radiografías simples en proyección anteroposterior de hombro y proyección tipo Zanca (Figura 2).

Las variables incluidas fueron edad, género, lado afectado, enfermedades asociadas, complicaciones postoperatorias tempranas (infección, dehiscencia de herida quirúrgica, hematoma, rechazo o desanclaje de material o pérdida de la reducción) y tardías (rechazo o desanclaje del material). Se evaluaron los resultados funcionales mediante la escala de evaluación de hombro UCLA. Esta escala, ampliamente 
validada, evalúa dolor, arcos de movilidad, fuerza, función y conformidad del paciente. Las puntuaciones van en el siguiente rango: de 0 a 20 puntos malo; de 21 a 27 regular; de 28 a 33 bueno; de 34 y 35 puntos excelente.

El seguimiento de los pacientes fue a 30, 90 y 365 días.

Descripción de la técnica quirúrgica. El paciente se coloca en posición silla de playa. Con asepsia y antisepsia del miembro torácico y del hemitórax afectados, se cubre con campos estériles. Se realiza infiltración del área a incidir con ropivacaína $5 \mathrm{~cm}^{3}$. Con incisión longitudinal a nivel de tercio distal de clavícula de $4 \mathrm{~cm}$, se diseca por planos hasta localizar la cara superior de la clavícula y el borde superior de la apófisis coracoides.

Bajo control fluoroscópico se hace perforación en borde superior de clavícula, con broca guía de $2.7 \mathrm{~mm}$ en dirección a la base de la apófisis coracoides. Posteriormente, se perfora con broca canulada $4.5 \mathrm{~mm}$. Se introduce guía flexible pasa hilos, con la cual se logra pasar el sistema de reducción a través del túnel, fijando de un ancla en la región inferior de la base de la apófisis coracoides y otra en la cara superior de la clavícula; la reducción se hace bajo visión directa y control fluoroscópico. Se cierra la herida por planos y después se coloca inmovilizador de hombro (Figura 3).

La técnica fue estandarizada por todos los ortopedistas participantes y la anestesia utilizada fue general balanceada.

La estadística utilizada fue descriptiva, medidas de tendencia central y de dispersión, correlaciones para variables independientes en el programa SPSS v. 22 de IBM.

\section{Resultados}

Fueron 17 pacientes con luxación acromioclavicular grado III. De éstos, 14 (82.4\%) fueron hombres y tres mujeres (17.6\%). La edad promedio de presentación fue 29.59 (de 19 a 44) \pm 7.475 años. Ningún paciente tuvo antecedentes personales patológicos positivos.

La presentación de la lesión en cuanto a género y lado afectado se muestra en la Tabla 1.

Figura 3:

Resultado de la reducción de la luxación acromioclavicular grado

III con sistema anclaje de doble botón.

\begin{tabular}{|c|c|c|c|c|}
\hline \multirow[b]{2}{*}{ Derecho } & & \multicolumn{2}{|c|}{ Hombro afectado } & \multirow[b]{2}{*}{ Total } \\
\hline & & Derecho & Izquierdo & \\
\hline \multirow[t]{2}{*}{ Género del paciente } & Hombre & 7 & 7 & 14 \\
\hline & Mujer & 2 & 1 & 3 \\
\hline Total & & 9 & 8 & 17 \\
\hline
\end{tabular}

\begin{tabular}{|c|c|c|c|}
\hline \multicolumn{4}{|c|}{$\begin{array}{l}\text { Tabla 2: Puntuación global obtenida de } \\
\text { la escala funcional de hombro. }\end{array}$} \\
\hline Paciente & 30 días & 90 días & 365 días \\
\hline 1 & 30 & 34 & 35 \\
\hline 2 & 28 & 34 & 35 \\
\hline 3 & 31 & 35 & 35 \\
\hline 4 & 29 & 35 & 35 \\
\hline 5 & 30 & 34 & 34 \\
\hline 6 & 31 & 35 & 35 \\
\hline 7 & 30 & 35 & 35 \\
\hline 8 & 29 & 35 & 35 \\
\hline 9 & 30 & 35 & 35 \\
\hline 10 & 32 & 35 & 35 \\
\hline 11 & 31 & 34 & 35 \\
\hline 12 & 28 & 35 & 35 \\
\hline 13 & 30 & 34 & 35 \\
\hline 14 & 31 & 35 & 35 \\
\hline 15 & 29 & 34 & 34 \\
\hline 16 & 30 & 35 & 35 \\
\hline 17 & 31 & 35 & 35 \\
\hline Media & 30 & 34.64 & 34.88 \\
\hline Mínima & 28 & 34 & 34 \\
\hline Máxima & 33 & 35 & 35 \\
\hline Desviación estándar & 1.11 & 0.49 & 0.33 \\
\hline
\end{tabular}

El tiempo promedio de la lesión a la fecha de la cirugía ambulatoria programada fue de 2.88 ( 1 a 7 ) \pm 2.147 días.

La evaluación funcional del hombro realizada en los tiempos distintos en todos los pacientes fue buena a los 30 días, y excelente a partir de los 90 y hasta los 365 días de operados. Los resultados se muestran en la Tabla 2.

Nadie presentó infección, dehiscencia de herida quirúrgica, pérdida de la reducción o desanclaje del material al año de seguimiento.

El tiempo medio de incapacidad otorgada a los pacientes fue 42.06 (de 28 a 56) \pm 7.894 días.

La correlación entre la edad y los días de incapacidad otorgados fue estadísticamente significativa $(\mathrm{p}=0.000)$ y se muestra en la Figura 4.

Ningún paciente presentó complicaciones tempranas ni tardías.

\section{Discusión}

La luxación acromioclavicular es una de las patologías más comúnmente diagnosticadas del hombro, como lo refie- 
re Rockwood desde 1984; predomina en gente joven y está asociada a actividades físicas o deportivas.

La literatura reporta los distintos grados de lesión, dependiendo de la deformidad y el desplazamiento de las estructuras que conforman esta articulación, es casi un hecho que las lesiones grado I y II de Rockwood se manejan mediante tratamiento conservador. ${ }^{1,2,3,4,5}$

La polémica en cuanto al manejo de las lesiones grado III siempre ha existido desde muchos años atrás, como lo comentan en su estudio Korsten y colaboradores, en una revisión sistemática, entre el manejo conservador y quirúrgico de la luxación acromioclavicular grado III, donde reportan que con los mismos trabajos utilizados por Smith TO y su equipo en el metaanálisis, además de agregar seis trabajos retrospectivos y comparar técnicas quirúrgicas distintas, implantes y tipos de inmovilizaciones, se concluye que la tendencia en Europa, con predominio en Alemania, es manejar la mayoría de estos pacientes quirúrgicamente, en contraste con el manejo conservador que predomina en Estados Unidos de América. ${ }^{6,7}$

En la literatura se encuentran varios estudios comparativos, entre métodos distintos de estabilización y el que se utilizó para este trabajo, como el realizado por Darabos y colaboradores, donde se compara la técnica quirúrgica entre el uso de un tornillo coracoclavicular y la colocación del TighRope (Arthrex Inc., Naples Florida USA), de este último se obtienen resultados funcionales y de satisfacción mejores en los pacientes operados. ${ }^{8}$

Vrgoc y colegas, en su estudio retrospectivo de los resultados entre el uso de alambre y sutura de forma abierta y la estabilización vía artroscópica mediante el uso del sistema TightRope (Arthrex Inc., Naples Florida USA), demostraron que el regreso a la actividad fue menor en los pacientes del grupo operados con alambre y sutura; sin embargo, el tiempo quirúrgico fue menor mediante la vía artroscópica, con resultados funcionales y estéticos mejores. Ambas técnicas

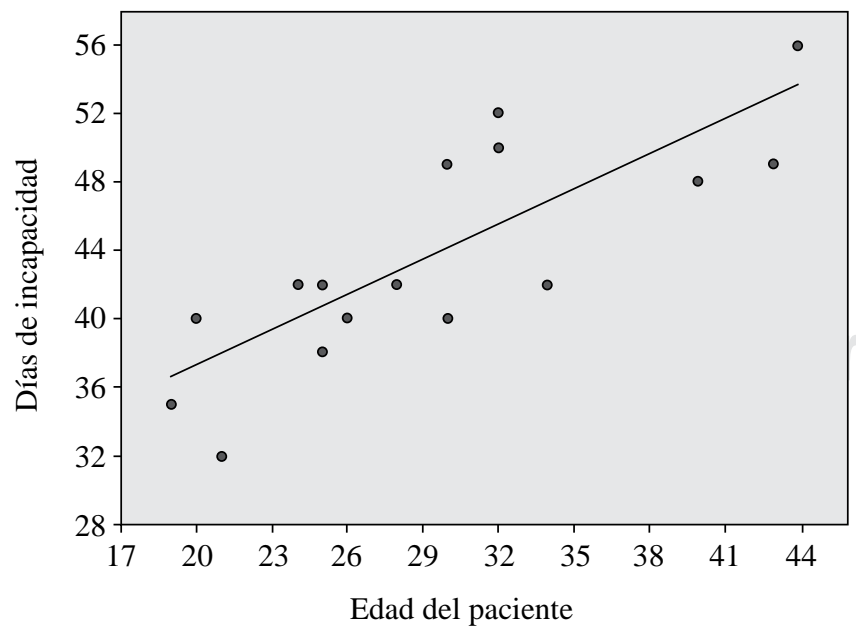

Correlación de Pearson $=0.796, \mathrm{p}=0.000$.

Figura 4: Correlación entre la edad y los días de incapacidad otorgados a los pacientes. ofrecieron resultados postquirúrgicos adecuados en las escalas funcionales aplicadas. El uso del sistema TightRope (Arthrex Inc., Naples Florida USA) fue 4.7 veces más caro que la técnica abierta, por lo cual se prefirió efectuarlo de forma abierta y prescindir del uso del artroscopio para evitar costos elevados. ${ }^{9}$

La Asociación Canadiense de Traumatología y Ortopedia, junto con Jeffrey Mah, realizaron un estudio multicéntrico del manejo conservador y quirúrgico mediante el uso de la placa gancho, y concluyen que ésta no ofrece resultados funcionales mejores como alternativa de manejo contra el tratamiento conservador. Compartimos dicha opinión, ya que se sabe que la estabilidad estática que proporciona una placa de este tipo no es la óptima para la reparación y estabilización de la luxación. ${ }^{10,11}$

Simon Lee y Asheesh Bedi describen el uso del implante que utilizamos en este estudio, entre otros, con buenos resultados. ${ }^{12}$

Chuanzhi X, Yaojia L y su equipo publicaron un artículo específicamente de los principios anatómicos para poder llevar a cabo la reducción de la lesión en forma dinámica, mediante técnica mínima invasiva con el uso de anclas, el cual usamos como referencia para la colocación del implante. ${ }^{13}$

Luis A Gómez y colegas publicaron un artículo acerca de la técnica artroscópica para la estabilización de la luxación acromioclavicular, mediante el uso del sistema TightRope (Arthrex Inc., Naples Florida USA) realizado en 10 pacientes, con un seguimiento de 12 a 15 meses de evolución, con edades de 26 a 42 años, muy similares a las de este estudio. Fueron valorados mediante la escala de UCLA con puntajes en promedio de 32.5, los cuales son satisfactorios, al igual que los obtenidos en este trabajo. ${ }^{14}$

Saier y colaboradores realizaron un trabajo de investigación para evaluar el retorno a la actividad posterior a la intervención quirúrgica, mediante las escalas DASH y Constant. Tomaron en cuenta el retorno a la actividad física parcial, completa y competitiva. En sus resultados demostraron que no hubo cambios en cuanto a su nivel de actividad funcional. En este estudio se observó un regreso a sus actividades laborales en todos los pacientes incluidos, sin complicaciones; no obstante, ninguno de los pacientes era deportista de alto nivel. . $^{15,16,17}$

En muchos centros hospitalarios aún continúan utilizando tratamiento conservador, ablativo como el Mumford y técnicas de estabilización estática. Nosotros consideramos que se deben emplear los recursos existentes como la técnica de reducción abierta, y estabilización dinámica mediante el uso de este sistema.

\section{Conclusiones}

Los resultados funcionales del tratamiento de la luxación acromioclavicular grado III con reducción abierta y fijación, a un año de seguimiento, son excelentes, mediante reducción abierta, y después de un año de la operación. El tiempo de incapacidad es corto y la reintegración a la actividad laboral es rápida. 
Bibliografía

1. Galatz LM, Williams GR. Lesiones de la articulación acromioclavicular. En: Rockwood \& Green's Fracturas en el adulto. Bucholz R, Heckman J, Rockwood C, Green D. Marbán. 5a ed.. Madrid, España, 2003. p. 1209-44.

2. Rockwood CA, Williams GR, Young DC. Trastornos en la articulación acromioclavicular. En: Hombro. Rockwood CA, Matsen FA, Wirth MA, Harryman DT. Marbán. 3a ed. Madrid, España, 2006. p. 479-550.

3. Gutiérrez-Meneses A, Iñárritu-Cervantes AS, Martínez-Molina OA. Luxación acromioclavicular. En: Gutiérrez-Meneses A, MartínezMolina OA, Valero-González FS. Patologías del hombro. Alfil. 2a ed. México D.F, México, 2010. p. 443-60.

4. Mansat M. Cirugía del hombro y del codo. Elsevier Masson. Barcelona, España, 2007. p. 92-100.

5. Kapandji IA. Fisiología articular. Tomo 1. Panamericana. 6a ed. Madrid, España, 2001. p. 58-61.

6. Korsten K, Gunning A.C, Leenen LP. Operative or Conservative Treatment in patients with Rockwood Type III acromioclavicular dislocation. A systematic review and update of current literature. International Orthopaedics. 2014; 38(4): 831-8.

7. Smith TO, Chester R, Pearse EO, Hing CB. Operative Versus no operative management following Rockwood Grade III acromicoclavicular separation: a meta-analysis of the current evidence base. J Orthopaed Traumatol. 2011; 12(1): 19-27.

8. Darabos N, Vlahovic I, Gusic N, Darabos A, Bakota B, Miklic D. Is AC Tight Rope fixation better than Bosworth screw fixation for minimally invasive operative treatment of Rockwood III AC joint injury? Injury. 2015; Suppl 6: S113-8. doi: 10.1016/j.injury.2015.10.060-
9. Vrgoc G, Japjec M, Jurina P, Gulan G, Jankovic S. Operative treatment of acute acromioclavicular dislocations Rockwood III and V-Comparative study between K-wires combined with FiberTape vs. Tight Rope System. Injury. 2015; 46S: S107-12.

10. Mah JM. Canadian Orthopaedic Trauma Society (COTS). General health status after non-operative versus operative treatment for acute, complete acromio-clavicular joint dislocation: results of a multicenter, randomized clinical trial. J Orthop Trauma. 2017; 31: 485-90.

11. Renfree KJ, Wright T. Anatomy and biomechanics of the acromioclavicular and esternoclavicular joints. Clin Sports Med. 2003; (22): 219-37.

12. Lee S, Bedi A. Shoulder acromioclavicular joint reconstruction and outcomes. Curr Rev Musculoskelet Med. 2016; 9: 368-77.

13. Chuanzhi X, Yaojia L, Qiang W, Gang C, Hansheng H, Zhihua L. Anatomical principles for minimally invasive reconstruction of the acromioclavicular joint with Anchors. Int Orthop. 2016; 40(11): 2317-24.

14. Gómez-Viera LA, Visco A, Daneu-Fernandes LF, Gómez-Cordero NG. Artrhoscopic treatment of acromioclavicular joint dislocation by tight rope technique (Arthrex ${ }^{\circledR}$ ). Rev Bras Ortop. 2009; 44(1): 52-6.

15. Saier T, Plath JE, Beitzel K, Minzlaff P, Feucht JM, Reuter S, et al. Return-to-activity after anatomical reconstruction of acute high-grade acromioclavicular separation. BMC Musculoskeletal Disorders. 2016; 17: $145-51$

16. Saccomanno MF, Milano G, De Ieso C. Acromioclavicular joint instability: anatomy biomechanics and evaluation. Joints. 2014; (2): 87-92.

17. Mazzocca AD, Spang JT, Rodriguez RR, Rios CG, Shea KP, Romeo AA, et al. Biomechanical and radiographic analysis of partial coracoclavicular ligament injuries. Am J Sports Med. 2008; 36(7): 1397-402.

Financiamiento: Ninguno. 